\title{
ZU DEN INSCHRIFTEN DES CLERMONTER RUNENKÄSTCHENS.
}

Der wettlauf von veröffentlichungen der skulpturen und inschriften dieses kästchens von den jahren 1900 und 1901 hat sich beruhigt und kein neuer gelehrter ist seither an den stoff herangetreten, um die zweifel zu lösen, die sich an die erklärungen, insbesondere der vierten, früher litterarisch nicht zugänglich gewesenen inschrift, durch Wadstein, Napier und Vietor heften mussten. Die deutschen anglisten schweigen.

Es wäre aber sicherlich unrichtig, die erklärung der vierten inschrift als aussichtslos auf sich beruhen zu lassen, da doch die wörter und verse der hauptsache nach feststehen und es vermutlich nur kleiner, unscheinbarer verschiebungen und änderungen bedarf, um einen in formen und satzfügung einwandfreien text $\mathrm{zu}$ erhalten.

In welchen genaueren beziehungen der erläuterung dieser text $\mathrm{zu}$ den skulpturen der betreffenden seitenwand stehe, was diese darstellen und ob für sie litterarische, geschichtliche oder sagenhafte, allesfalls auch mythologische überlieferungen aufgespürt werden können, die geeignet sind ihren inhalt deutlich vor augen zu stellen, das ist nun freilich eine sache für sich.

Es ist Napiers verdienst die inschrift, die Wadstein in drei selbständige teile zerfällt hatte, als éinen zusammengehörigen text von drei langversen erkannt zu haben, so wenigstens s. 17, wogegen er später allerdings wieder zweifel erhebt; zugleich hat er die lesung der drei, oder wie Wadstein meinte vier letzten runen im rechten seitenbande in entscheidender weise festgestellt: konjunktion swá, kein adj., am allerwenigsten ein solches zu vier zeichen. Ferner verdanken wir ihm die erkenntnis der metrischen beschaffenheit des zweiten 
halbverses swá hivi ertae ziszráf, sowie die deutung von ertae als subst. im nom. (nach seiner meinung ein personenname) und von ziszráf als $3 \mathrm{sg}$. praet. ind. zu zescrífan 'iudicare, assignare, imponere, designare'.

Indem Napier des weiteren die von Wadstein zuerst fälschlich für genitive plur. gehaltenen, dann in seiner zweiten veröffentlichung auf eine mehrheit von flexivischen möglichkeiten zurückgeführten singularischen casus sorzoe und tornoe als instrumentale fasst, hat er sich die grundlage für eine gerundete übersetzung der inschrift geschaffen: 'here hos sits on the sorrow-hill, endures tribulation as Ertae had imposed upon her, rendered wretched by sorrow and anguish of heart', die denn auch der gegenständlichen beziehung zum rechten abschnitte des mittelfeldes: frau bei einem grabhügel sitzend, nicht entbehrte.

Die betrachtungen, die ich im folgenden an diese deutung knüpfe, nehmen ihren ausgang von der formellen unwahrscheinlichkeit der Napier'schen erklärung des komplexes sarden als ${ }^{*} s \overline{\bar{e}} r d \overline{\bar{o}} n={ }^{*} s \bar{a} r$ zid̄ōn 'rendered wretched' und führen von diesem punkte aus zu einer, wie ich hoffe der wahrheit genauer entsprechenden darstellung des syntaktischen bildes.

Zur lesung der inschrift möchte ich zunächst bemerken, dass es mir nicht erlaubt scheint, die gewöhnlichen ags. runen $e M$ und $a K$ in den komplexen scerden im unteren längenbande und sefa im linken seitenbande nur aus dém grunde mit anderen werten: $w$ und $u$ nach Napier, zu lesen, dass die vokale $e$ und $a$ an den übrigen stellen der inschrift mit den besonderen zeichen $x$ und $h$ dargestellt würden, über deren mögliche herkunft ich Z. f. d. Phil. 33, 409 ff. ausführlich gehandelt habe. Ich muss vielmehr hinsichtlich der geltung der beiden zeichen an dem a. a. o. gesagten festhalten. Der runenschneider, der für die vokale der umschrift der vierten kästchenseite besondere vokalzeichen gebraucht, dagegen in den paar wörtern des mittelfeldes sich ebenso wie an allen übrigen inschriftstellen des kästchens der gewöhnlichen ags. vokalrunen bedient, hat im komplexe scerlen das gewöhnliche ags. $M$ gesetzt, weil ihm der unmittelbare zusammentritt seines besonderen $e x$ mit seinem $\mathrm{zu}$ diesem im verhältnis des vertikalen spiegelbildes stehenden geneigten $n \times$ aus ästhetischen gründen, oder wahrscheinlich noch mehr aus gründen der graphischen deutlichkeit 
unerwünscht war. Er hat ferner in der ligatur $\widehat{f a}$ mit den seitenästen des $f$ an der oberen, denen des $a$ an der unteren rechten hälfte der gemeinsamen vertikalen hasta, sich wiederum des gewöhnlichen $₹$ bedient, da diese ligatur eine aus der kenntnis des ags. runenalphabetes verständliche, vielleicht auch sonst gebräuchliche war, eine ligatur des $f$ mit dem besonderen $a$ der vierten inschriftseite aber schwer verständlich erscheinen musste. Dass er aber überhaupt eine ligatur setzte, wird in diesem falle doch wohl auf gründen der platzverteilung beruhen; die betrachtung des linken seitenbandes lehrt ja sofort, dass innerhalb des rahmens von gegebener grösse eine elfte selbständige rune nicht untergebracht werden könnte.

Auf der festhaltung einer lesung $\widehat{f}$, die ich a. a. 0 . vorgeschlagen habe, beharre ich nicht angesichts der bestimmten versicherung Wadsteins Z. f. d. Phil. 34, 127, dass die ligatur nicht das seitendetail des $\mathbb{E}$, sondern das des $F$ enthalte, dass das bild der inschrift auf tafel 6 bei Napier in diesem punkte täusche und durch einen in seinen händen befindlichen abdruck berichtigt werde.

Ich halte es ferner gegenwärtig nicht für thunlich, die restierende aufrechte hasta nach azl am ende des oberen längenbandes so $\mathrm{zu}$ ergänzen, dass eine ags. entsprechung $\mathrm{zu}$ dem belegten got. adjektivabstractum aglo, oder zu einem $\mathrm{zu}$ mutmassenden got. *aglei derselben wortkategorie sich ergäbe. Eine endung $a$ oder $e$, die mit den besonderen runenzeichen der umschrift dargestellt wäre, ist durch die dastehende gradlinige und senkrecht orientierte hasta aus graphischen gründen ausgeschlossen, eine endung $u$ oder $o$ allerdings möglich, doch wieder deshalb nicht gerade wahrscheinlich, weil ursprüngliche adjektivabstracta auf $-u$ (ig. $\bar{a}$ ), oder auf $-\infty$, später $u$ (ig. $-\bar{o} n$ ), im ags. nicht vorzukommen scheinen, eine abstraktbildung auf got. - $e i$, ags. sekundär -o aber umlaut des stammvokales erforderte. Es wäre also *ezlo (: adj. ezle) zu erwarten, wofür allerdings *azlo, *azlu, nicht aber *azlo, -u geschrieben sein könnte. Napier wollte daher das $a$ des wortes als 'miscut' für $a$ angesehen wissen. Das gewicht der thatsächlichen belege $\mathrm{zu}$ ags. azlác dréozan 'to suffer misery, torment' bei Bosworth-Toller 1, 29 ist indessen so stark, dass man sich zur annahme gedrängt sieht, die aufrechte hasta als rest einer ligatur von $K$ und $h$ zu betrachten, in die, wie bei der 
ligatur $\hat{f a}$, die gewöhnlichen ags. runen eingetreten sein werden. Diese annahme trägt sowohl der behauptung Napiers rechnung, dass in dem zur verfügung stehenden raume bis zur abschliessenden leiste eine zweite rune nicht untergebracht werden kann, als auch anderseits der entgegenstehenden lexikalischen wahrscheinlichkeit, die trotzdem für das bekannte, von Wadstein gefundene wort azlác spricht.

Meine in Z. f. d. Phil. vertretene auffassung des einganges der umschrift her hos sitch als weibl. personenname *Herhós + verbum gründete sich darauf, dass im mittelfelde der skulptur nur éine figur dargestellt ist, die man für eine weibliche halten kann; das kopftuch, das sie trägt, gleicht wenigstens dem der weiblichen gestalt im offenen fenster der deckelskulptur des kästchens und auch der stabartige gegenstand, den sie in gesichtshöhe vor sich hält, ist ihr mit dieser figur gemeinsam. Dass diese weibliche gestalt der rechten seitenwand aber sitzend dargestellt sei, möchte ich nicht mit sicherheit behaupten, obwohl für stehende stellung der untere teil der gestalt stark verkürzt erschiene.

Da man indessen das verbum sittan der umschrift nicht wörtlich zu nehmen braucht, sondern auch als 'verweilen, sich aufhalten' verstehen kann, so ergiebt sich aus der darstellung kein grund, der gegen die verbindung der weiblichen figur mit dem herhos des textes spräche. Ich habe also die eventualität eines personennamens in den vordergrund gestellt, weil, wenn hér das örtliche adv. und hós das ags. appellativum wäre, die skulptur nicht klappte, man nähme denn an, dass eine mehrheit, eine 'cohors' auch durch éine figur dargestellt werden könne. Die analogie der legende der rückseite des kästchens, eingeleitet mit her feztap Titus ..., ist aber allerdings ein beachtenswerter grund, von einem personennamen abzusehen und her hōs sitcep als 'hīc cohors sedet' zu verstehen, um so mehr, als der eingang mit hër überhaupt beliebt ist. Temporales $h \bar{e} r$ 'at this time' leitet in der Parkerhs. der Chronik (Kluge, Leseb. 38 ff.) die einzelnen abschnitte ein, wie hér fénz Aberéd ... tó Wesseaxna ríce (z. j. 866), hér rád sé here eft tó Eoforicicceastie ... (\%. j. 870), gleichbedeutend mit einleitendem $b a ́$, wie jua féng AElfréd AElelwulfing ... tó Wesseaxna ríce, mit wortfolge: adv., praedikat, subjekt. Die wortfolge: adv., subjekt, praedikat, die in hér hós sitcp vorläge, 
hat daselbst gleichfalls ihre parallele in hér hópne men árest on Sceápize ofer winter sátun (z. jahre 855).

Die verschiedenheit der endung des verbums in sitap und drizip kann man in zweifacher weise aufklären. Entweder enthält drizip die ältere northumbr. endung von zescéadip, cymid, wyrcid, die nach Sievers $\S 358$ durchweg in Ep., zu $2 / 3$ (gegen $1 / 3 e$ ) in Corp. herrscht, dann ist das -cep von sitce $p$ eine gelegentliche entlehnung aus der germ. $a i$-konjugation, ahd. $\bar{e}$, und schliesst sich an north. haefep, haefeep in $\mathrm{R}^{1}$ : habban, got. habaip, ahd. hapêt, oder es steht sitcep gleich dómoed in Ps. für *site $\not$, dessen $\breve{e}$ jüngere vertretung für $\breve{\imath}$ ist, was allerdings Napier s. 14 für die zeit des denkmals, anfang des 8. jahrh. nach seiner meinung, für unmöglich hält, dann enthält drizip am ehesten orthographische auflösung izi für gesprochenes $\bar{\imath}$ in ${ }^{*} d r \bar{\imath} p$ aus ${ }^{*} d r i u z i p$, wozu sich die, vermutlich mit zweigipfligem accent gesprochenen tid und flid $<{ }^{*} t i u h i p$, *fliuhip (Siev. § 165, 2) vergleichen.

Hinsichtlich des vokales im ersten teile des kompos. hermberze 'sorrow-hill' zu ags. hearm glaubte Napier, es sei vielmehr harm zu erwarten und der runenmeister habe wohl einen fehler begangen, indem er $c e$ für richtiges $a$ zur darstellung brachte. Aber northumbr. $a$ für $e a$ vor $r$ ist nicht ausnahmslose regel, es erscheint daneben zuweilen auch $a$ Siev. $§ 158$, ebenso Ep. zwei fälle mit ae : faerh, maerh, so dass wir uns wohl mit dieser gelegentlichen lautgebung für sonstige brechung ea abfinden können. Da nun ags. beorh in der that vom 'grabhügel, tumulus' gebraucht wird, darf man *hearmbeorh, hoermberz wohl als poetische bezeichnung desselben ansehen, die sich in ihrem bestimmungsworte wie hearmloca 'peinigungsort', von der hölle und vom gefängnis gesagt, verhält. Ist aber hoermberz das im mittelfelde abgebildete hügelgrab, so wird on nicht als 'auf', sondern als 'bei' gleich ct 'apud' verstanden werden müssen, was u. a. durch den gebrauch der konjunktion bei ortsnamen wie on Hripum u. a. beleuchtet wird. Es muss aber wohl hervorgehoben werden, dass diese auffassung nicht die allein mögliche sei, dass hormberz auch der name der lokalität im allgemeinen sein könnte, also ein berg, auf dem sich das abgebildete grab samt der hós befindet.

Swae hiri ertae ziszráf ist relativsatz, anknüpfend an 
azlác 'miseria'. Der parallelismus dieses satzes zu dem von Napier aus Beow. 2574 verglichenen swá him wyrd ne zescráf hréd oet hilde ist allerdings nicht vollkommen, da wir es an dieser stelle mit einem kausalsatze 'da ihm die Wyrd keinen erfolg im kampfe bescheerte' zu thun haben. Aber der dativ des persönlichen pronomens findet sich hier wie in Caedm. 3067-69 se de him ... zescráf wéan ... in ähnlicher position.

Der flexionsvokal des dativs sing. des persönl. pronomens fem. hiri, wofür wir *hirce erwarten, ist eine entlehnung aus der nominalen $\breve{o}$-deklination und verhält sich wie in den fem. dativen sing. ródi und coestri. Ertae ist das subjekt des relativsatzes und steht für *erpce, ws. eorpe swf. 'terra'. Die form unserer inschrift zeigt abgesehen von der vertretung der brechung eo durch einfaches $e$, wozu man Ep. herth, ferth, ferred, sifunsterri (Dieter s. 20) vergleiche, die in den älteren quellen vorkommende getrennte schreibung -ae für - $a$ (Napier s. 15 note), wie Ep. thohae 'argillus', clatae 'blitum', ribbae 'canis lingua', ferner jene schreibung der tonlosen spirans $p$ mit $t$, die gleichfalls eine eigentümlichkeit der älteren ags. orthographie ist, wie auslautend feormat 'fouit', siftit 'crebrat', neben anhriosith 'ingruerit' Ep., oder inlautend tha deatlicostan 'funestissima' ebenda, Sútborne 'Southbourn' Kemble cod. diplom., Sútangli Urk. (Siev. § 199 a. 1).

Die wiedergabe von $s c r$ mit $s z r$ in ziszraf wollte Napier s. 16 a. 1 als verlegenheitsschreibung erklären. Der schreiber habe die beiden ags. $c$-runen, d.i. die mit dem geradlinigen und die mit dem gebrochenen unten an die aufrechte hasta gelehnten seitenbalken, für seine besonderen vokalzeichen $\boldsymbol{x}$ und $a$ verbraucht und musste daher für $c$ nach einem ersatz suchen, den er in dem lautlich naheliegenden $z$ fand. Das ist nun allerdings nicht ganz richtig, den die $c$ in fisc stirnseite, costri linke seite und risci mittelfeld der rechten seite besitzen ja eine ordnungsgemäss senkrecht auf die grundlinie einsetzende haupthasta und einen ungebrochenen seitenstab, während bei dem besonderen $x$-zeichen unserer inschrift die haupthasta den boden gar nicht erreicht, sondern im zweiten drittel über der grundlinie sich symmetrisch gabelt und das seitendetail des besonderen $a$ ein gebrochener schrägstab ist, aber immerhin mag zugegeben werden, dass die graphische scheidung des $c$ und der besonderen zeichen für $a$ und $a$ in 
unserer inschrift nicht den wünschenswerten grad der klarheit und entschiedenheit besitzt. Der grund für die schreibung szr liegt aber trotzdem kaum in einem graphischen bedürfnis, sondern in den thatsachen der lautbildung, die für scr eine mittlere fortis $c$ bedingen, deren abstand vom verschlusslaut $g$ ein so geringer ist, dass sie mit dem zeichen für diesen wiedergegeben werden kann. Die in zweiter und dritter silbe erscheinenden ahd. $s g$-schreibungen für $s c$, wie arsgeidan, drisguflim, pisgerito, farthrosgan, ganaozsgaffi (Kögel, Üb. das Keron. Glossar s. 91) gehören in den kreis dieser lauterscheinung, die im vorliegenden falle durch das folgende $r$ nur gesteigert werden konnte.

Scerden, das neben twœzen und afœddde der linken seiteninschrift unmöglich entrundetes $e<\infty$ haben kann, erkläre ich als swf. partizipium sérde und apposition zu hós, $\left.{ }^{1}\right)$ in der aussprache verschmolzen mit der präposition in (dieselbe auch auf der linken seite des kästchens), $\mathrm{zu}$ der die dative sorza und sefatornce gehören. Wir sollten nach dem stande der endsilben unseres denkmales ${ }^{*}$ scerdoe gewärtigen, es hat also, da wir sárde ' $n$ für ${ }^{*}$ sárdae in vorfinden, das anlautende $i$ der präposition auf den auslaut des voranstehenden partizipiums umlautend eingewirkt. ${ }^{*} S e ́ r d c e$ ist natürlich nicht von dem intransitiven ags. sárian, -ode 'to feel pain for', ahd. sêrôn 'dolere', sondern von dem transitiven verbum ahd. sêren 'uulnerare', wozu auch as. gisêrid, afries. sêrd gehören, abgeleitet, das auch im ags. umlaut haben und *scéran lauten müsste. *Sórde nimmt also das subjekt hós mit einem anderen ausdrucke wieder auf und (i)n sorzce and sefatornce fügt eine modale bestimmung hinzu. Das kompos. sefatorn enthält wahrscheinlich den $n$-losen northumbrischen genitiv von sefa. Im zusammenhange übersetzt lautet die inschrift, wenn wir hós in der that nach dem dativ mazpa hóse 'ancillarum turbae' Béow. 924, got. hansa, ahd. hansa 'cohors' appellativisch fassen: 'hic cohors sedet ad tumulum sepulcralem, miseriam patitur, quam ei terra imposuit, exagitata illa in sollicitudine et cordis tribulatione'. Die häufung der trauerwörter kann gegen die wahrscheinlichkeit dieser auffassung nicht geltend gemacht werden, da z. b. in Caedm. 75 sár and

1) Vgl. zum swf. Kudrun 1703,4 des bin ich ... diu sorgen vrîe. 
sorze, súsl prówedon sogar drei, nahezu identische ausdrücke für den begriff des leides als objekt in éiner langzeile stehen.

Metrisch verhält sich hēr hốs sítap etwa wie Hel. 362, 1 an érdágon, ebenso on lıármbérza; ázlāc drîzip wie Hel. 219, 1 hêtan scóldi oder 413, 1 lófuuord mánag und sárde 'n sórzce wie Hel. 196, 1 bárn an búrgun (Mon.). Dem relativsatz swäe hiri ërpae ziszräf vergleicht sich Hel. 2459, 2 endi an grämono hróm oder 2298, 2 unas im bötono thärf (Mon.), endlich dem halbvers and séfatórnce Hel. 275, 2 fan hëtanuuánge.

Hinsichtlich der skulptur des mittelfeldes möchte ich darauf hinweisen, dass dieselbe vielleicht christlichen inhaltes sei und auf die schlussszenen des leidensdramas Christi bezug habe.

Der grabhügel könnte Christi grab sein und die bei demselben befindliche weibliche gestalt eine der trauernden frauen nach Matth. 27, 61 'erat autem ibi Maria Magdalene et altera Maria sedentes contra sepulchrum'. Das wort risci könnte man nach ags. risce 'iuncus, scirpus' als 'arundo' fassen und auf das 'rohr' beziehen, mit dem Christus von den römischen soldaten verhöhnt und geschlagen wird Matth. 27, 29 .. p0suerunt ... et arundinem in dextera eius ... 27, 30 'et ... acceperunt arundinem et percutiebant caput eius'.

Das reittier im mittelfelde könnte man mit dem eselsfüllen identifizieren, auf dem Jesus fünf tage vor pascha sich nach Jerusalem auf den weg macht Joh. 12,14 'et inuenit Jesus asellum, et sedit super eum ...' und das wort wudu könnte als 'lignum' verstanden auf das kreuzesholz bezogen werden.

Im besonderen beachtenswert wären unter dem gesichtspunkte dieser auffassung der kelch oder becher und das wort bita rechts oben im mittelfelde. Beide könnte man auf die einsetzung des abendmahles beziehen und zwar bita 'frustum, buccella' auf das gebrochene brot Matth. 26, 26 'accepit Jesus panem ... ac fregit deditque discipulis suis', den becher auf Matth. 26, 27 'et accipiens calicem ... dedit illis ...', doch scheint es mir ebenso möglich das wort bita als übersetzung. des im Johannesevangelium genannten ausdruckes 'buccella' zu betrachten 13, 27 'et post buccellam ...' und 13, 30 'cum ergo accepisset ille buccellam ...', der sich auf den dem verräter Judas von Jesu gereichten, eingetauchten bissen bezieht, 
so dass bild und wort in der auffassung des künstlers vielleicht nach beiden seiten bezogen wurde.

Ich füge auch $\mathrm{zu}$ den übrigen inschriften des kästchens einige neue bemerkungen hinzu.

Der name des bogenschützen auf der deckelplatte $A E_{z}$ ili ist $i o$-stamm mit altem $i$ des nominativs wie mask. heri, endi, neutr. ríci und deckt sich mit westfränk. Aglius episcopus 696 bei Pardessus Diplomata nr. 437, deutsch Egili Piper Libri confrat. II 423. Er ist kurzform oder patronymikon aus einem mit agila-, ags. azel- zusammengesetzten zweistämmigen namen und in den ortsnamen Ezelesbyriz, Ezlesford, Ezleslona, AEzeleswurd, heute einheitlich Ayles-bury, -ford, -lane, -worth enthalten, wozu auch die alten kontraktionen EEles-beorh, -ford (alle formen bei Kemble Cod. dipl. VI) gehören werden. Schon Binz in PBB 20, 190 notiert diese namen nebst anderen hierzu, aber ursprünglichen diphthong in der stammsilbe anzunehmen, hat er sich wohl gehütet; der etymologische ansatz ags. * Ëzili $=$ hd. Eigil ist Wadsteins und Viëtors eigentum, und er ist falsch. Dass allerdings die hd. kontaminationsform Eigil nicht bloss hd. und schon älteren datums sei, beweisen westfränk. Aiglus z. j. 693 bei Pardessus, ags. Aighelestorp (cit. bei Binz a. a. 0.) und ahd. Aigil St. P.; den lautlichen vorgang, dem sie ihr zustandekommen verdankt, kann man auch als sekundäre neuauflösung des synkopierten *aila- betrachten, ein besonderes namenselement, das von *agila- verschieden wäre, ist aber in ihr nicht gegeben.

Dass die mit der überschrift hronces $b \bar{a} n$ 'os ceti' eingeleitete legende der stirnseite metrisch sei, ist bekannt.

Es vergleicht sich zur ersten halbzeile der beiden langverse

1. físc flódu ahtf on fërzenbériz;

2. warp żásric zrórn, pāer he on zrëut ziswóm.

der Heliandvers 2910, 1 náht néflu biuuárp, zum zweiten halbvers: Hel. 1491, 2 an hëlligruind, 1506, 2 an lêtthan uuég, oder mit berücksichtigung des swarabhaktivokales in beriz etwa Hel.2133, 2 Mon. an hëbenrîki, auch 275,2 fan hëbanuuánge. Der ersten hälfte des zweiten langverses entspricht, nicht ganz genau Hel. 2634, 1 an uuídan uuíg, der zweiten Hel. 2730, 2 huo hie gifüodit unás oder 2379, 2 obar that thëgno fólc, auch 2401, 2 Mon. endi eft üp gigéng. 
Was ferzenberiz betrifft, dessen erster teil bei Kemble Cod. diplom. einmal in der obliquen form Ferezenne als ortsname nachgewiesen ist, entscheide ich mich jetzt dafür, womit Viëtor einverstanden sein wird, den ersten teil hier überhaupt nicht im sinne von 'berg', sondern nach ahd. Virgundia waldus 814 Kausler, Wirtemb. Urkdb. in dem von 'wald' zu nehmen, so dass für das kompos. der sinn 'waldhöhe' sich ergiebt und die frage, inwieweit dieses appellativum topisch fixiert $\mathrm{zu}$ denken sei, füglich bei seite gelassen werden kann.

An der lesung zasric ist nichts zu ändern. Die zwei übereinander gestellten punkte : im innern des wortes zwischen den runen $a$ und $s$ füllen nur den leeren raum, nicht anders wie der éine punkt hinter fisc und der hinter flodu. Sie sind ornamental, nicht graphisch zu beurteilen und können weder als trennungs- noch als verweisungszeichen verstanden werden.

Das wort wurde von Sweet in Engl. Studien 2, $314 \mathrm{f}$. als ältere form des ausdruckes zársecz 'ocean' erklärt, der mit metathese $s r>r s$ und umdeutung auf zár und secz (: ags. secz 'salum, mare') zu stande gekommen wäre. $\mathrm{Zu} z \bar{a} s$ hielt er an. geisa 'to chafe, rage', zur ableitung ahd. wuoterich 'tyrannus'.

Diese ahd. bildung aber, wozu auch andd. wûdrich 'truculentus', mhd. sudrich 'koch', die sich an die personennamen auf -rîh anschliesst, ist ein persönlicher, kein sächlicher terminus und man müsste demnach zäsric als namen des persönlich gedachten oceans fassen, so dass der verdacht einer mythologischen personifikation sehr nahe gerückt wird. In diesem falle könnte aber in das kompos. älteren datums, dessen ersten teil wir genauer mit an. geis f. 'voldsom fremfærd' (ahd. vielleicht in den hydrographischen namen Geismari, Geisaha, Geisbach) bestimmen können, der zweite teil rric wohl noch

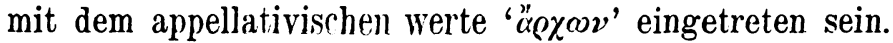

Dass man, wie Sweet glaubt, zrorn nicht in der belegten, auch durch as. grornōn bestätigten bedeutung 'maestus', sondern als 'turbidus' verstehen müsse, finde ich nicht einleuchtend, da dem persönlich gedachten flutbeherrscher wohl auch trauer um einen verlornen wal zugeschrieben werden kann und pár würde man in diesem zusammenhange doch wohl eher temporal 'cum', als lokal 'dort wo' aufzufassen haben.

Die umschrift der stirnseite, deren zweiten teil wir übersetzen wollen: 'piscem fluctus sustnlit in montem siluaticum; 
Gasricus maerore affectus est cum in litus natauit', macht sowohl das material aus dem das kästchen gefertigt ist namhaft, als auch die gelegenheit, bei der das material gewonnen wurde. Die skuJptur im rechten felde stellt die adoratio der magi ab oriente dar (z. b. Matth. 2, $1 \mathrm{ff}$.), wobei zu beachten, dass die runische überschrift der szene mazi nicht bloss im stammvokal nostrifiziert, sondern auch hinsichtlich der flexion als ags. nom. pl. der $i$-deklination zu denken ist. Die skulptur im linken felde mit dem kunstreichen schmied Wieland als hauptfigur, ist aber ohne zweifel als eine art gewerblichen emblemes des künstlers, der das kästchen verfertigte, gemeint.

Weniger anerkannt ist die metrische natur der linken seiteninschrift :

1. Rómwalus and Réumwalus twì̄̇en zibrópcer;

2. af ⿳亠丷厂犬dda hice wýlif in Rómoecóstri,

3. ópla únnēz.

wie denn auch zugestanden werden muss, dass die verteilung der liedstäbe in diesen versen einiges $\mathbf{z u}$ wünschen übrig lässt. Reguläre allitteration zeigen nur 1,1 und 3,1 , während 1,2 und 2 der allitteration entbehren. Es ist übrigens interessant, dass 1,1 und 2,2 herausgehoben sich zu einem vollkommenen allitterationsvers verbänden, so dass man $\mathrm{zu}$ dem schlusse veranlasst wird, es seien hier die zwei langverse 1 und 2 hinsichtlich der allitteration als teile eines grösseren metrischen ganzen $\mathrm{zu}$ betrachten.

Metrisch vergleicht sich zu 1: Hel. 1256 Andrease endi Pétruse érist sáne Cott. und der hierauf folgende halbvers 1257, 1 gibrüother tuéna würde im *tuena gibruother umgestellt, sich nicht nur in den wörtern, sondern auch metrisch mit dem ags. halbverse 1,2 vollkommen decken.

Wer die verse 2 und 3,1 für teile éines langverses hielte, könnte den ersten teil leicht aus Hel. 2777, 2, mit elision gelesen: thuo sia it gihotrdun thia mágat sprecan Cott., tho sie that gihordun thea mágad sprekan Mon. verifizieren, doch wird es sich empfehlen, diese zusammenlegung von 2 und 3, 1 nicht zu vollziehen. $\mathrm{Zu} \mathrm{2,1}$ halte man Hel. 1232, 1 alêthian thèm liudun, zu 2, 2: Hel. 61, 1 an Híerusálem, zum ganzen verse Hel. 1297 Críst alouuáldo gicóran hábda Cott. Der halbvers 3 er- 
läutert sich aus Hel. 3570, 1 hlüdo hréopun, 3546, 1 uurêthan uuilleon, 3544, 1 uuilleon uuíson.

Rommalus und Reumvalus geben sich sofort als lateinisierungen eines ags. paares ${ }^{*}$ Romvvalh und ${ }^{*}$ Reumwalh zu erkennen, dessen auslautendes $h$ vor der latein. endsilbe nach analogie der gedeckten obliqui des ags. deklinierten wortes behandelt, d. h. synkopiert ist.

Diese umbildungen der beiden römischen namen müssen natürlich der intern ags. überlieferung angehören und sind auf grund von älteren entlehnungen ${ }^{*} \operatorname{Romul}(u)$ und ${ }^{*} \operatorname{Reumul}(u)$ nach der kategorie der ags. personennamen auf -walh wie Adilualch, Ealdwealh, Cónualh geformt. Für *Rōmwalh wird insbesondere noch der ags. volksname *Rōmwalas 'Römer', belegt gen. reht Rōmwala 'ius Quiritum' Rit. eccles. Dunelmens., dat. mid Rūmwalum 'apud Romanos' Wídsíđ 69, hereinspielen, der sich an die deutschen, sekundär determinierten ortsnamen Strâzuualaha, Trûnuualha, Cûrunuala, dat. Cûrouualahon anschliesst und eine ags. parallele in Bretwalas 'die Kelten in Wales' besitzt. Lat. Rèmulus erscheint sowohl gleichbedeutend mit Rèmus bei Sulpic. sat. 19 und ist in diesem falle sicher eine formelle angleichung an Rōmulus, als auch bei Ovid als name eines königs in Alba, bei Vergil als name eines helden. Es ist wahrscheinlich, dass diese erweiterte form für Reumwalus vorauszusetzen sei, obwohl der $u$-umlaut ebenso gut aus einfachem *Reumu < Rĕmus erklärt werden und die sekundäre umformung dieses zu einem kompos. mit -walh, -walus sehr wohl auch als ags. analogiebildung nach dem ersten namen des brüderpaares gefasst werden kann. Da Remi nepotes bei Catull soviel wie 'Römer' ist, kann auch für Reum-walus die bedeutung des volksnamens mit in frage kommen. Die umschrift von germ. walh in latein. -ualus findet sich auch auf dem kontinent, so westfränk. Bernoalus 9. jh. Pol. Irm.

Das kompos. *Römcecester enthält gleich dem mit anderem zusammengesetzten Rómeburz den gen. sing. des stadtnamens ags. Róm f., ebenso as. Rümuburg z. b. Hel. 57 den gen. eines stf. ${ }^{*} R \bar{u} m a$, erschlossen zu Hel. 3809. Ags. Rómaburh aber enthält wohl einen nach analogie der volksnamen eingetretenen gen. pluralis.

Völlig neu ist meine metrische auffassung der inschrift der rückseite des kästchens, für die ich vorausschicken muss, dass sie allerdings die von mir Z. f. d. Phil. bd. 23 vorge- 
schlagene ergänzung ziupea-su[nu] zur voraussetzung hat, dass sie aber zugleich rückwirkend geeignet ist, die richtigkeit dieser ergänzung zu bewähren.

Die zwei langverse

1. Hēr féztap Tìtus end ziứpeasú[nu];

2. Hīc fúgiănt Hiếrŭsălĭm ắfitātơrēs.

zu denen die unterschrift des kästchens $D \bar{D} m$-zisl ${ }^{1}$ ) natürlich in keinerlei inneren textlichen beziehungen steht, sind vom standpunkte der kunstmässigen allitterationspoesie allerdings wieder sehr unvollkommen, da sie jeder für sich betrachtet keinerlei liedstäbe zeigen. Man muss sie ebenfalls hinsichtlich der allitteration als eine einheit fassen, um die beabsichtigte anwendung des kunstmittels in feztap, fugiant einerseits und żiupea, Hierusalim anderseits zu sehen. Wir werden die zweite bindung, zu der man Hel. 788 that sie thar te Hierusalem Iudeo liudi Mon. halte, als hauptallitteration des verspaares betrachten müssen.

Fugiant in *fugiunt zu korrigieren und für afitatores, das eine aktuelle aussprache *avitatores zur darstellung bringt, die korrekte latein. schreibung habitatores herzustellen, unterlasse ich. Man muss nur wissen, dass das dastehende latein. verbum fugiunt sein soll und dass das $h$ in hierusalem, wo es im sinne der latein. orthographie gesetzt ist, ebenso stumm ist, als in afitatores, wo es gegen die latein. orthographie weggelassen erscheint.

$\mathrm{Zu} \mathrm{1,1}$ halte man Hel. 837, 1 thurh hlútran huigi Mon., zu 1,2: Hel. 2648, 2 that gödes barn séhan, zum ganzen verse Hel. 75 Jácobas súneas, guiodero thiedo, auch 74 uuás fan them líudeon Léuias cúnnes.

2,1 kann man in Hel. 612, 1 so huát so an Hiérusalem wiederfinden, selbst wenn man mit elision *suat so liest. Auch der vorcitierte halbvers 788,1 , mit elision *thát $s$ thar te Hiérusalem gelesen, entspricht im allgemeinen. Den typus von 2, 2 aber stellen sowohl die unmittelbar zugehörigen halbverse 612, 2 gúodara mánno Cott. und genauer 788, 2 Júdeo líudi dar, als auch die wegen ihrer inhaltlichen und grammatischen be-

1) Der zweite teil des namens, ursprünglich mit lang $\bar{\imath}$ anzusetzen, kann hier schon gekürzten vokal haben. 
ZU DEN INSCHRIFTEN DES CLERMONTER RUNENKÄSTCHENS. 449

ziehungen zu dem uneigentlichen, mit dem gen. pl. des volksnamens Judeas gleich sonstigem Judeapéod gebildeten kompositum Jiupeasu[nu] im besonderen, beachtenswerten Hel.halbverse 104, 2 Hébreo líndi, 307, 2 Ëbreo fólces, 766, 2 Júdeono fólkes.

Bemerkenswert ist die form der in völlig tonloser position stehenden konjunktion end gegen sonstiges and der kästcheninschriften. 1) Der umlaut derselben ist vermutlich hier durch das anlautende $i$ des folgenden wortes bedingt, zu dem die konjunktion im verhältnis der proklise steht. Hierusalim ist

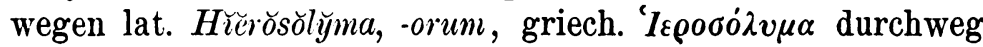
mit kurzvokalen anzusetzen. In unserm text erscheint das wort als indeklinabile mit der funktion eines genitivs.

1) Vgl auch Ep. 2 f. 23 aend, Corpus 17, 9 end.

WiEN, 24. 10. 03.

von Grienberger. 